\title{
Physical Estimation of X-Ray Triplet Phases in a Centrosymmetric, Mosaic Crystal with Unit Cell Volume $\sim 3000 \AA^{3}$
}

\author{
Frode Mo, ${ }^{a}$ Bjørn C. Hauback ${ }^{a}$ and Gunnar Thorkildsen ${ }^{b}$
}

${ }^{\text {aInstitutt for røntgenteknikk, Universitetet i Trondheim-NTH, N-7034 Trondheim-NTH and }{ }^{\mathrm{b}} \mathrm{H} ø g s k o l e s e n t e r e t} \mathrm{i}$ Rogaland, Ullandhaug, N-4000 Stavanger, Norway

\begin{abstract}
Mo, F., Hauback, B. C. and Thorkildsen, G., 1988. Physical Estimation of X-Ray Triplet Phases in a Centrosymmetric, Mosaic Crystal with Unit Cell Volume $\sim 3000 \AA^{3}$. - Acta Chem. Scand., Ser. A 42: 130-138.

$\mathrm{X}$-ray phases have been determined physically from three-beam interactions in a centrosymmetric, mosaic crystal with unit cell volume $\sim 3000 \AA^{3}$. A brief review of theoretical conditions and a discussion of some important practical consequences for such experiments are given, along with a discussion of the procedure employed in the present work. A four-circle diffractometer set up for data collection with filtered MoKa radiation was used for the measurements. 22 interaction maxima were examined by repeated $\omega / 2 \theta$ scans over the primary diffracted intensity while rotating $\psi$ in steps over the exact three-beam position. Phases could be assigned to 17 triplets ( $77 \%$ of total), among them 7 negative, from careful analyses of the intensity profiles mapped as a function of $s_{L}$, the signed distance of the secondary reciprocal lattice point from the Ewald sphere. They were used later as input in a direct-method program to solve the unknown structure.

The results demonstrate that this method has greater potential than has been realized before. Phase measurements for crystals with considerably larger cells should be within reach, certainly under better optimized experimental conditions. Some advantages of the method in crystal-structure work are discussed. One potentially interesting area is that of structures possessing pseudo-translation symmetry, which can be exceedingly difficult to solve by standard crystallographic methods.
\end{abstract}

It has been known for more than 30 years that information on X-ray phases can be retrieved from multiple-beam ( $n$-beam) diffraction experiments with perfect crystals. Experimental evidence was obtained first with electrons, ${ }^{1}$ and later with X-rays. ${ }^{2}$

More recently it has been realized that phase effects can be significant also in mosaic crystals. However, most crystals studied in the past were apparently of fairly high perfection, and possible mosaic character was not assessed (see e.g. Ref. 3). We reported our first successful phase measurements in $1982 .{ }^{4}$ They appear to be among the earliest measurements made with truly mosaic crystals, in this case of two centrosymmetric, organic structures, viz. 1,2-bis(methylsulfonyl)ethane (MMDS, $\mathrm{C}_{4} \mathrm{H}_{10} \mathrm{O}_{4} \mathrm{~S}_{2}$ ) and 1,2-bis(phenylsulfonyl)ethane (PPDS, $\mathrm{C}_{14} \mathrm{H}_{14} \mathrm{O}_{4} \mathrm{~S}_{2}$ ). Both speci- mens had mosaic spread $\gamma \sim 0.06-0.08^{\circ}$, the diffracted intensity profiles being Gaussian with full width at half maximum (FWHM) 0.15$0.18^{\circ}$ in $\theta$. We subsequently measured phases from a crystal of dithiooxamide (DTO, $\mathrm{C}_{2} \mathrm{H}_{4} \mathrm{~N}_{2} \mathrm{~S}_{2}$ ) which had intensity profiles of Lorentzian type with FWHM $\sim 0.23^{\circ}$ in $\theta .^{5}$

These and other experiments reported in the literature (see e.g., Refs. 6 and 7) demonstrate that structure-factor phase information can be extracted under $n$-beam diffraction conditions from crystals of moderate mosaic character and with relatively small unit cells. The results are interesting and important. However, from an applied point of view, there was little evidence until now that this physical method offers a real alternative to other techniques for solving the phase problem in crystallography. Its possible

130 Acta Chemica Scandinavica A 42 (1988) 130-138 
usefulness in crystallographic work is limited by several factors: line-broadening factors, which include crystal mosaicity and size and the angular and spectral distributions of the incident beam, volume of the unit cell, intensity and wavelength of the incident beam, non-centrosymmetry, etc. It is necessary to explore the potential of the method by repeating the measurements with crystals which constitute more demanding tests. Keeping in mind the applied aspect, it is also of great interest to examine what can be done with standard diffraction equipment and radiation sources. In practice, this means working under non-optimal experimental conditions.

We present here results obtained from phase measurements on a mosaic, centrosymmetric organic crystal with unit cell volume $\sim 3000 \AA^{3}$. The experiments were carried out at $86 \mathrm{~K}$ with a standard four-circle diffractometer set up for data collection, and a $\mathrm{Nb}$-filtered beam of Mo radiation from a fine-focus $\mathrm{X}$-ray tube. A preliminary presentation of our experimental procedure and the main results has been given ${ }^{8}$ (see also Ref. 9).

\section{Theory}

In the following we review briefly the theoretical background of immediate relevance to the discussion of the experimental strategy and interpretation of the results.

\section{A. Approximate solution of the fundamental} equation. The wavefield created in a crystal when $n$ beams interact under mutual exchange of energy can be described by the Takagi-Taupin equations or by the fundamental equation of the plane-wave dynamical diffraction theory. The former set of equations has been used in studies of three-beam diffraction in perfect, infinite ${ }^{10}$ and finite $^{11}$ crystals. The present discussion is based on the fundamental equation of the plane-wave theory. Adopting the formalism of Batterman and Cole $^{12}$ we obtain eqn. (1) [cf. their eqn. (A12)]. Here, $\mathbf{k}$ and $\mathbf{K}(\mathbf{H})$ are wave vectors, with $k$ along the incident beam (vacuum) and $\mathbf{K}(\mathbf{H})$ along the primary diffracted beam (crystal), and $|\mathbf{K}|=|\mathbf{k}|[1-\Gamma F(\mathbf{O})]^{1 / 2} ; \mathbf{E}(\mathbf{H})$ is the electric field vector corresponding to vector $\mathbf{K}(\mathbf{H}), \Gamma=$ $r_{\mathrm{e}} \lambda^{2} / \pi V$, where $r_{\mathrm{e}}$ is the classical electron radius, $\lambda$ is the wavelength and $V$ is the volume of the unit cell; $F(\mathbf{H})$ is the structure factor of reflection $H$.

The solution of eqn. (1) for two beams $(n=2)$ is treated in the literature (see, e.g., Refs. 12 and 13). For $n \geq 3$ the full solution of (1) must be carried out numerically, and the influence of the various parameters is then no longer easily traceable. Analytical solutions for these cases involve approximations. In one approach, three-beam diffraction $(n=3)$ is treated as a perturbed twobeam case. The method is analogous to one introduced by Bethe ${ }^{14}$ in electron diffraction. In this model only the incident, $\mathbf{O}$, and primary diffracted, $\mathbf{H}$, beams are assumed to be strongly coupled. Secondary reciprocal-lattice points (r.l.p.'s) are near, but not on the Ewald sphere. Therefore, coupling to the corresponding beams is weak, and they are treated within the kinematic approximation. In the X-ray case, a second simplifying approximation is to neglect terms which connect waves with different directions of polarization. The Bethe approximation has been worked out for X-ray diffraction in a perfect crystal by Marthinsen, ${ }^{15}$ who also examined the validity range for some of the assumptions involved.

We have developed a first-order approximation to the solution of (1) for the symmetrical Laue case, using as perturbation parameter $s_{L}$, the distance of the secondary r.l.p. $L$ from the Ewald sphere. We have studied the effects on both the real and imaginary parts of the structure factor, as well as the influence of line-broadening factors on the asymmetry features in the primary diffracted intensity. The study forms part of a Doctoral thesis, ${ }^{16}$ and some of the results have already been presented. ${ }^{5} \mathrm{~A}$ full description of this work, including a second-order approximation which is not based on Bethe's model, will be the subject of a forthcoming publication. ${ }^{17}$

For the present discussion absorption effects can be neglected since $\mu t<0.1 ; \mu$ is the linear absorption coefficient, $t$ is the average crystal thickness. Hümmer and Billy ${ }^{18}$ have shown that for $\mu t<0.5$, absorption effects make no significant contributions to the phase-related asymmetry in the diffracted intensity profiles. Therefore, we give here only the first-order approximation for the real part of the structure factor. For a perfect crystal in the vicinity of a three-beam

$$
\left[k^{2}(1-\Gamma F(\mathbf{O}))-K^{2}(\mathbf{H})\right] \mathbf{E}(\mathbf{H})-k^{2} \Gamma \sum_{\mathbf{L} \neq \mathbf{H}} F(\mathbf{H}-\mathbf{L}) \mathbf{E}(\mathbf{L})+[\mathbf{K}(\mathbf{H}) \cdot \mathbf{E}(\mathbf{H})] \mathbf{K}(\mathbf{H})=0
$$




$$
\begin{aligned}
& I(\mathbf{H}) \propto|F(\mathbf{H})|^{2} p_{O H}^{2}\left[1-k \Gamma \frac{|F(\mathbf{H}-\mathbf{L})||F(\mathbf{L})|}{|F(\mathbf{H})|} \cdot \frac{\left(p_{O L}\right)\left(p_{H L}\right)}{\left(p_{O H}\right)} \cdot \frac{\cos (\varphi(\mathbf{H})+\varphi(\mathbf{L}-\mathbf{H})+\varphi(-\mathbf{L}))}{s_{L}}\right. \\
& \left.+\left(\frac{1}{2} k \Gamma \frac{|F(\mathbf{H}-\mathbf{L})||F(\mathbf{L})|}{|F(\mathbf{H})|} \cdot \frac{\left(p_{O L}\right)\left(p_{H L}\right)}{\left(p_{O H}\right)} \cdot \frac{1}{s_{L}}\right)^{2}\right] \\
& I(\mathbf{H}) \propto Z|F(\mathbf{H})|^{2}\left[1-2 T^{1 / 2} P R_{F}\left(\cos \Phi_{3} / s_{L}\right)+T P^{2} R_{F}^{2}\left(1 / s_{L}\right)^{2}\right]
\end{aligned}
$$

point the integrated intensity is given by (2). Here, $p_{O H}$ is the polarization correction for scattering of beam $\mathbf{O}$ into $\mathbf{H}, s_{L}$ is def. $>0$ for $L$ outside the sphere, and $\varphi(\mathbf{H})+\varphi(\mathbf{L}-\mathbf{H})+\varphi(-\mathbf{L})$ is a three-phase structure invariant (SI). Introducing: $T=1 / 4 k^{2} \Gamma^{2}, Z=\left(p_{O H}\right)^{2}, P=\left(p_{O L}\right)\left(p_{H L}\right) /$ $\left(p_{O H}\right), R_{F}=|F(\mathbf{H}-\mathbf{L})||F(\mathbf{L})||| F(\mathbf{H}) \mid$ and $\Phi_{3}=$ $\varphi(\mathbf{H})+\varphi(\mathbf{L}-\mathbf{H})+\varphi(-\mathbf{L})$, one obtains (3) (see Ref. $5)$ which has the same form as deduced by Juretschke $^{19}$ for the symmetrical Bragg case (see also Refs. 20 and 21 ).

The terms in square parentheses can be regarded as the dynamical correction of the twobeam intensity developed within the limits of the Bethe approximation. It is the second term that carries information on phase, and more specifically on the value of a three-phase SI, $\Phi_{3}$, in the case of three interacting beams. We have shown that this term appears in a different analytical form when line-broadening factors are considered. Its asymptotic properties, however, are very similar to those of the corresponding term in (3). ${ }^{5}$ The phase term retains its form also in higher-order approximations. Hence, (3) can be used to illustrate in a very simple manner how an observed asymmetry in the tails of the primarydiffracted intensity profile is related to an unknown three-phase SI through the known parameter $s_{L}$. A plot of (3) for three different values of $\Phi_{3}$ is shown in Fig. 1. Here, $y_{B}$ represents the terms in square parentheses in (3), i.e. $I(\mathbf{H}) \propto$ $Z|F(\mathbf{H})|^{2} y_{B}$, and $x_{s}=s_{L} /\left(T^{1 / 2} P R_{F}\right)$, thus

$$
y_{B}=1-2 \cos \Phi_{3} / x_{s}+1 / x_{s}^{2}
$$

cf. Juretschke. ${ }^{19 b}$ For $\Phi_{3}=0, \pi$, the two tails of the intensity distribution approach the two-beam level from opposite sides as $\left|s_{L}\right|$ increases. The asymmetry vanishes for $\Phi_{3}= \pm \pi / 2$ when $\cos \Phi_{3}=0$. For a perfect crystal the most pro- nounced asymmetry is found for $s_{L}$ of the order of $10^{-7}-10^{-6} \AA^{-1}$.

Expr. (3) is developed under the assumption that the secondary r.l.p. $L$ does not lie on the Ewald sphere, i.e. $s_{L} \neq 0$. In fact, (3) diverges for $s_{L} \rightarrow 0$, and cannot be used to estimate $I(\mathbf{H})$ at, or very close to, the three-beam point. This is not a serious defect, however, since we are interested here in the intensity distribution away from this point.

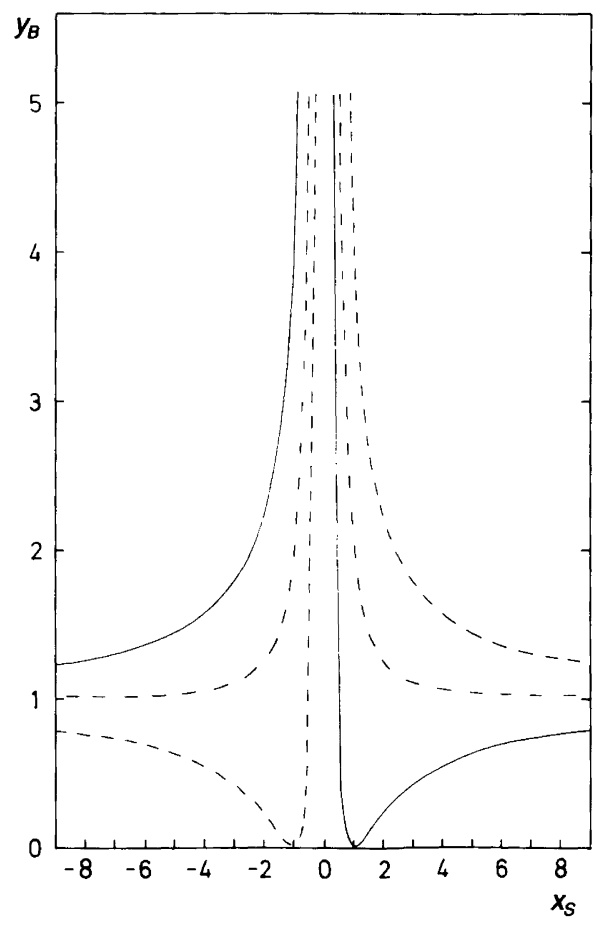

Fig. 1. Dynamical correction, $y_{B}$ [eqn. (4)], of a primary diffracted intensity near a three-beam point calculated by the Bethe approximation. The curve for $\Phi_{3}= \pm \pi / 2$ has been included for completeness. $\Phi_{3}=0:-; \Phi_{3}=\pi / 2:-\cdot-\cdot-; \Phi_{3}=\pi:---$. 
In the phase term of (3), $T^{1 / 2}=1 / 2 k \Gamma$ is an experimental constant, of the order of $10^{-9} \AA^{-1}$ for $\lambda=\lambda(\operatorname{Mo} K \bar{\alpha})$ and for $V=3000 \AA^{3} ; P \sim 1$. Thus, the magnitude of the asymmetry ratio $M_{I}=I(\mathbf{H})$ $\left(s_{L}<0\right) / I(\mathbf{H})\left(s_{L}>0\right)$ for a given crystal is determined primarily by the parameter $R_{F}=|F(\mathbf{L})| \mid F$ $(\mathbf{H}-\mathbf{L}|/| F(\mathbf{H}) \mid$. The most favourable case appears to be when $|F(\mathbf{H})|$ is small, and both $|F(\mathbf{L})|$ and $|F(\mathbf{H}-\mathbf{L})|$ are large (however, see Experimental strategy and measurements).

B. Line-broadening factors. Several factors associated with the nature of real crystals and radiation sources will effect broadening of the diffraction lines. In particular, crystal mosaicity is a severely limiting parameter. The width of the $n$-beam perturbation peaks or troughs increase as a result of experimental convolution, and the sharp features of the profile become smeared and partially disguised under the peak or trough. What is left for observation is a greatly reduced asymmetry further away from the $n$-beam point. ${ }^{17}$ For a crystal of given size and mosaicity the experimental conditions would be improved by decreasing both the angular divergence (focusing) and the bandwidth (monochromatization) of the incident beam. These points have been emphasized in particular by Ladel1 ${ }^{22}$ and by Post and collaborators. ${ }^{23}$ The favourable properties of synchroton radiation for this type of work were demonstrated recently by Chang. ${ }^{24}$

C. Integrated power of the diffracted beam. A preferable and often mandatory condition for these experiments is that both the secondary, $L$, and coupling, $H-L$, reflections, should be strong as measured from the crystal. Thus, factors that in general reduce the integrated power, $P(\mathbf{H})$, of the crystal, also become limiting for our purpose. Assuming kinematic diffraction in the imperfect crystal, one has: ${ }^{25}$

$P(\mathbf{H})=I(\mathbf{O}) r_{e}^{2}|F(\mathbf{H})|^{2} \frac{V_{x}}{V^{2}} \lambda^{3} \frac{p_{O H}^{2}}{\sin 2 \theta}$.

New quantities are: $I(\mathbf{O})=$ intensity of the incident beam, and $V_{x}=$ volume of the crystal. For a given crystal size and primary reflection $H$, (5) shows that the integrated power increases with increasing $I(0)$ and $\lambda$, and decreasing $V$. The expectation value for $|F(\mathbf{H})|^{2}$ is given by:
$\left.<|F(\mathbf{H})|^{2}\right\rangle=\sum_{j=1}^{n} f_{j, H}^{2}$,

where $f_{j}$ is the scattering factor of atom $j$, and $n$ is the number of atoms per unit cell. Assuming a roughly constant crystal density, then $n \propto V$ and $\left\langle|F(\mathbf{H})|^{2}\right\rangle \propto V$. One may therefore write $P(\mathbf{H}) \propto V^{m}$ where $m$ is closer to -1 than to -2 as in (5).

Absorption is not taken into account in (5). Between absorption edges, the mass absorption coefficient, $\mu_{m}$, for an element varies with the wavelength as $\mu_{m} \propto \lambda^{q}$, and $q \sim 2.75$ for a large range of elements when $\lambda<\lambda(K$-edge) and $\lambda(K$ edge $)<\lambda<\lambda\left(L_{\mathrm{l}}\right.$-edge $){ }^{26} \mathrm{~A}$ simple calculation taking into consideration both the integrated power and absorption ${ }^{25}$ gives an optimum crystal thickness of the order of $1 / \mu$. In single-crystal work the size of the specimen is limited by the two-dimensional intensity distribution of the incident beam. With a fine-focus X-ray tube the maximum dimension is usually in the range 0.8$1.0 \mathrm{~mm}$. For most organic compounds without heavy atoms $\mu<1 \mathrm{~mm}^{-1}$ for $\mathrm{Cu} K \bar{\alpha}$ radiation, and so the optimum thickness is not attained in practice. This is no longer true when the crystal contains more strongly absorbing elements.

In the general case, changes will take place in both the real (dispersion) and imaginary (absorption) parts of the structure factor under $n$-beam diffraction conditions, and the combined effects determine the asymmetry. These points will be discussed in more detail elsewhere.

D. Frequency of n-beam situations. For a crystal in random orientation, the number of r.l.p.'s lying within a shell of thickness $2 \Delta$ about the Ewald sphere is:

$N \sim 8 \pi \Delta\left(V / \lambda^{2}\right)$

$N$ in eqn. (6) includes so-called "extinct" reflections due to space-group symmetry. Insofar as various kinds of deviations from the simple, spherically symmetric model for the bonded atom affect the space-group symmetry, the concept of "extinction" loses its exact meaning. Therefore, these reflections may be treated along with those that happen to be near zero as the result of a particular distribution of electron density in the asymmetric unit. Very weak primary reflections 
$H$ are particularly sensitive to $n$-beam interactions, cf. the factor $R_{F}$ in (3). Only in those cases where all interacting reflections are very weak can the transfer of energy between the various diffracted beams be neglected.

Most organic, mosaic crystals that were used in our experiments had diffraction maxima with FWHM $\sim 0.15-0.18^{\circ}$ in $\theta$. We found that secondary r.l.p.'s within about $5 \cdot 10^{-4} \AA^{-1}(=\Delta)$ from the Ewald sphere could give rise to significant intensity perturbations. For crystals with broader intensity profiles, like DTO, the critical value of $\Delta$ will be larger. Setting $\Delta=5 \cdot 10^{-4} \AA^{-1}, V=$ $400 \AA^{3}$ and $\lambda=0.71073 \AA$, one obtains $N \sim 10$. Eqn. (6) shows how $N$ increases with increasing $V$ and decreasing $\lambda$. Changing the radiation from MoKō to $\mathrm{Cu} K \bar{\alpha}$ reduces $N$ by a factor of nearly 5 .

The results show that the number of closely spaced $n$-beam situations is large even for a crystal with a small unit cell. In general, only a small fraction of them have $R_{F}$ values sufficiently large to produce measurable phase asymmetry. Significant changes in peak intensity, in particular of the Umweg type, occur more frequently, and this problem seems to be underestimated in current accurate crystallographic work.

Clearly, both the wavelength and the unit cell volume are critical parameters in experimental phase measurements. The optimal wavelength represents a compromise between several, in part counteracting factors discussed in Sections $B, C$ and $D$. In our studies of organic crystals we have used $M o K \alpha$ radiation, but for the crystals with the largest unit cells, $\mathrm{CuK \alpha _{1 }}$ or radiation of even longer wavelength, would be more favourable.

For the great majority of crystals that have been subjected to phase measurements, the unit cell was relatively small. The largest observed until the present work seems to be that of the intermetallic compound $\mathrm{Cs}_{10} \mathrm{Ga}_{6} \mathrm{Se}_{14},{ }^{6,27}$ with $V \sim$ $2160 \AA^{3}$. The unit cell volumes for the crystals studied previously by us were in the range $\sim 233$ (DTO) to $684 \AA^{3}$ (PPDS).

\section{Experimental}

Crystal data and instrumentation. A crystal of an organic compound (EHM III) with unknown structure and assumed chemical composition $\mathrm{C}_{18} \mathrm{H}_{15} \mathrm{ON}_{3}$ was used for the experiment. The unit cell was monoclinic, space group $I 2 / a$, with $a=$ 17.820(1), $b=14.857(1), c=11.299(1) \AA, \beta=$ $99.17(1)^{\circ}, V=2953.2(4) \AA^{3}$ at $86 \mathrm{~K}$, and $Z=8$. A complete data set had been collected previously for a different specimen out to $2 \theta_{\max }=60^{\circ}$, or $(\sin \theta / \lambda)_{\max }=0.704 \AA^{-1}$ with Nb-filtered MoK $\bar{\alpha}$ radiation. In all experiments, the cell parameters and orientation matrix were calculated from the setting angles of the $K \alpha_{1}$ peaks of 20 reflections in the $2 \theta$-range $55-61^{\circ}\left[\lambda\left(\mathrm{MoK} \alpha_{1}\right)=\right.$ $0.70930 \AA]$. The centring procedure was repeated several times during the work. All profile measurements were carried out with a four-circle Picker FACS-I diffractometer and $\mathrm{Nb}$-filtered $\operatorname{Mo} K \alpha$ radiation $[\lambda(\operatorname{Mo} K \bar{\alpha})=0.71073 \AA]$, the angular divergence of the incident beam being $0.12^{\circ}$. The profiles of the diffracted intensities from this crystal had FWHM $\sim 0.15^{\circ}$ in $\theta$, and the crystal edges were $\leq 0.5 \mathrm{~mm}$.

Experimental strategy and measurements. Intensity profiles for the experimental estimation of triplet phases were obtained from $\psi$ scans: ${ }^{28}$ The intensity of a primary diffracted beam is measured as a secondary r.l.p. $L$ is rotated about the r.l. vector $\mathbf{H}$ through the Ewald sphere. Stepscans are superior to continuous scans for mapping out profiles, the latter method being inadequate when the signal/background ratio becomes small. We have previously compared profiles from stationary measurements vs. scans. In the scan-mode the integrated intensity is measured in an $\omega / 2 \theta$ - or $\omega$-scan for each little step in $\psi$. $^{5}$ Stationary measurements are more critically affected than scans by small errors in the orientation matrix. We found the scan technique generally preferable, in particular for small values of the asymmetry ratio $M_{l}$. All intensity measurements for the EHM III crystal were made with $\omega / 2 \theta$ scans. Steps in $\psi$ were $0.02^{\circ}$ in the range $\psi_{0}-0.36$ to $\psi_{0}+0.36^{\circ}$, and $0.04^{\circ}$ further away; $\psi_{0}$ corresponds to the exact three-beam position. The total range in $\psi$ was $\psi_{0}-\Delta \psi$ to $\psi_{0}+\Delta \psi$, with $\Delta \psi$ usually $0.50^{\circ}$ although in some cases larger.

For mosaic crystals with relatively small unit cells, like that of PPDS with $V \sim 684 \AA^{3}$, phase asymmetry is easily observed also with strong primary reflections, $H$, provided that the secondary, $L$, and coupling, $H-L$, reflections, are both strong. ${ }^{4}$ In the phase-carrying term in (3), 
$T \propto V^{-2}$, and from the discussion in Section C, $R_{F}$ is approximately $\propto V^{1 / 2}$. Therefore, the product $T^{1 / 2} \cdot R_{F}$ is approximately $\propto V^{-1 / 2}$. Other parameters being constant, this implies that as $V$ increases, a smaller fraction of the possible threebeam (or $n$-beam) situations can effect a significant phase asymmetry in the primary diffracted intensity. It becomes necessary then to maximize the ratio $R_{F}$ by employing strong reflections for the secondary and coupling beams. There is another rationale for this strategy also: Because of their greatly enhanced sensitivity to $n$-beam effects, the intensity profiles of very weak $H$ reflections often may be a composite of several, in part overlapping, $n$-beam interactions. In our experience, therefore, very weak reflections are not well suited for phase measurements. Except for special purposes, we do not want $F(\mathbf{H})$ to be too small in order to obtain a more robust phase "transducer". In the present case we selected the 21 strongest reflections with $2 \theta<30^{\circ}$. Together with their symmetry equivalents, they comprised a group of 78 reflections with integrated peak counts (IPC) in the range $8 \cdot 10^{4}-9.2 \cdot 10^{5}$. Reflections $L$ and $H-L$ were required to belong to this group for all triplets. A second criterion for selection was that these reflections should participate in many $\Sigma_{2}$ relations. The primary reflections, $H$, that were examined had IPC's in the range $\sim 2.9 \cdot 10^{3}-9.8 \cdot 10^{4}$, the corresponding range of scaled structure factor amplitudes, $|F(\mathbf{H})|$, being 10.9-116.3, with an average of 54.6.

All reflections that were involved in our triplets had $2 \theta<30^{\circ}$. In view of the low angle values and the experimental parameters given previously, the broadening and the possible asymmetry introduced in the line profiles by using the unresolved $K \alpha$ doublet will be negligible. Within the given limit in $2 \theta$, certain regions of reciprocal space are inaccessible due to instrumental constraints. The constraints were included as boundary values for the angles $\omega$ and $\chi$ during a computer-search for possible triplets.

The profiles of 22 three-beam Umweg peaks were measured. In all cases we had checked that there were no other strong $n$-beam interactions in the vicinity of the peak being studied. For this purpose, all r.l.p.'s lying within a specified distance from the Ewald sphere were identified for each three-beam point by a method described by Tanaka and Saito. ${ }^{29}$

\section{Results}

Fig. 2 shows four of the experimental intensity profiles. The rotation in $\psi(\mathrm{deg}$.) has been transformed into $s_{L}\left(\AA^{-1}\right)$, the perturbation parameter. This quantity is of direct relevance, since the observed asymmetry, which is linked to the term with $s_{L}^{-1}$, defines the value of $\Phi_{3}$ in the centrosymmetric case without ambiguity. The four profiles in Fig. 2 correspond to the following three-beam interactions: (a) $44 \overline{2} / 0 \overline{4} 2 / \overline{4} 00$, (b) $233 / \overline{4} 00 / 2 \overline{3} \overline{3}$, (c) $332 / \overline{1} \overline{3} \overline{2} / \overline{2} 00$ and (d) $4 \overline{2} \overline{2} / 033 / \overline{4} \overline{1} \overline{1}$. Each point in a profile shows the average value from two or three, in some cases four, $\omega / 2 \theta$ scans made to improve the statistical accuracy. The error bar in each figure represents the e.s.d. in one point.

The phase effect on an intensity profile is recognized by differences both in the levels of the two backgrounds and in the slopes of the peak itself, the change in $\partial I / \partial s_{L}$ being larger on the low-background side. We have used the asymmetry in the background tails as a quantitative measure of phase. It is necessary first to examine whether the profile under study may be perturbed by other close-lying $n$-beam interactions. There are no significant secondary interactions in the cases (a), (b) and (c). In (d), however, two significant three-beam interactions appear in the vicinity of $\psi_{0}$ : one, at $\psi=-21.91^{\circ}$, is $4 \overline{2} \overline{2} / \overline{2} 33 / \overline{2} \overline{1} \overline{1}$, for which we calculated an intensity change of $+2 \%$; another is at $\psi=-21.69^{\circ}$, $4 \overline{2} \overline{2} / \overline{\overline{7}} \overline{1} 0 / 332$, with a calculated intensity shift $+0.9 \%$. The positions are marked with the shorter arrows in Fig. 2 (d). The relatively large variations in the tails of profile (c) cannot be ascribed to secondary $n$-beam interactions. We have observed similar and stronger fluctuations in other profiles. They seem to be dynamic effects, probably related to the particular geometrical conditions of these $n$-beam situations.

For the profiles (a) and (b) in Fig. 2 we used from 10 to 16 points, corresponding to 25-30 measurements, to calculate the weighted mean, $\bar{I}$, and error, $\sigma(\bar{I})$, for each background level. In both cases, the difference between the two, $\Delta \bar{I}$, is about $10 \overline{\sigma(I)}$, and $\bar{I}\left(s_{L}<0\right)>\bar{I}\left(s_{L}>0\right)$ shows that $\Phi_{3}=0$ (positive triplet). For profile (c), each background was defined by about 20 measurements, the ratio $\Delta \bar{I} / \overline{\sigma(I)}$ being 8 . Because of the close-lying three-beam interactions in case (d), only 5 points were used to define the background 

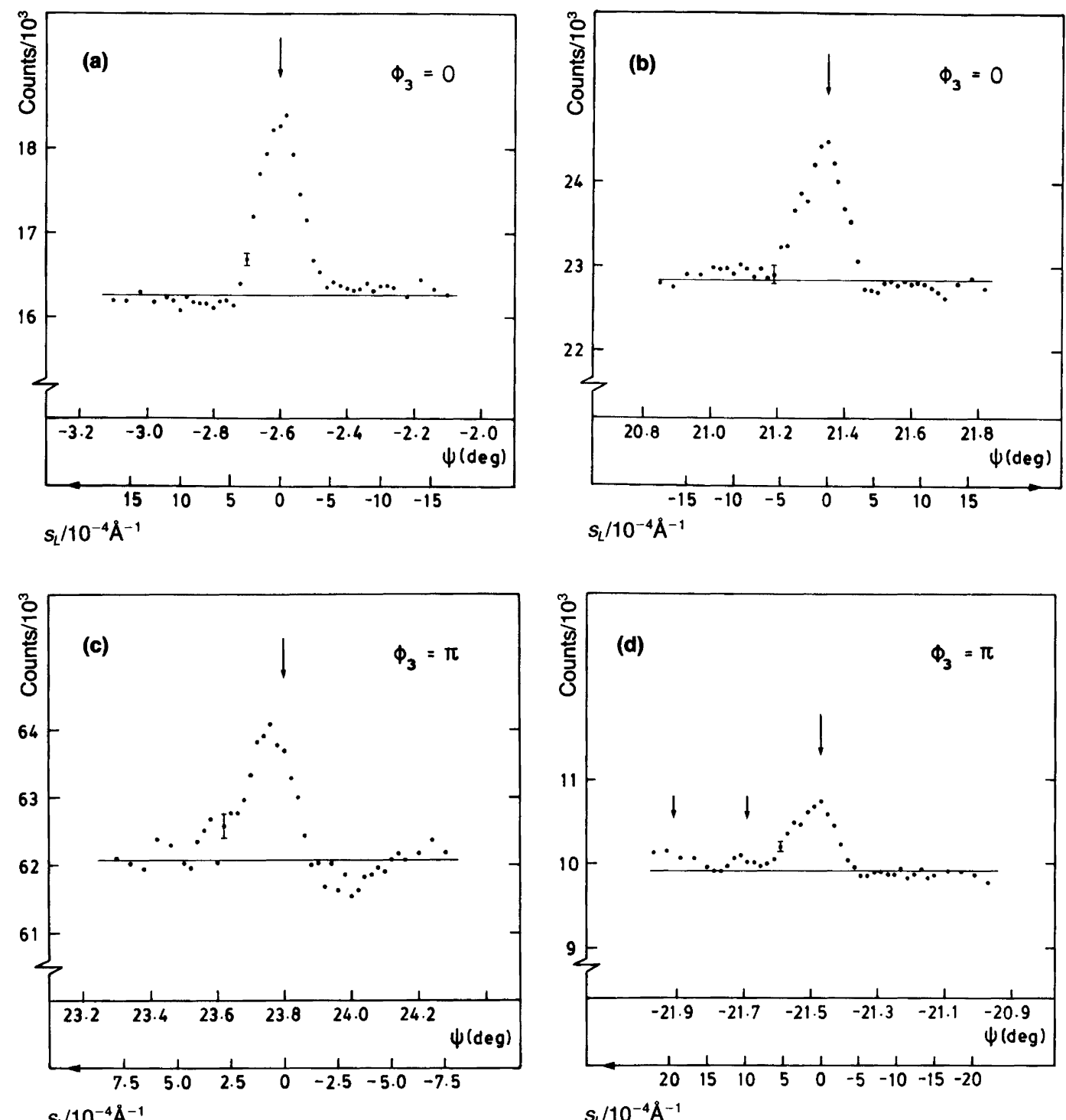

$S_{L} / 10^{-4} \AA^{-1}$

$S_{L} / 10^{-4} \AA^{-1}$

Fig. 2. Observed profiles of the primary diffracted intensity in four different three-beam interactions. Integrated intensity measured by $\omega / 2 \theta$ scans at fixed points in $\psi$ is shown also as a function of $s_{L}$, the excitation error of the secondary r.l.p. L. Error bars represent the e.s.d. in one point. In each plot, the calculated three-beam point, $s_{L}=0$, is indicated by the central arrow. In (d), two shorter arrows indicate secondary three-beam interactions. The triplets are: (a) $44 \overline{2} / 0 \overline{4} 2 / \overline{4} 00$, (b) $233 / \overline{4} 00 / 2 \overline{3} \overline{3}$, (c) $332 / \overline{1} \overline{3} \overline{2} / \overline{2} 00$ and (d) $4 \overline{2} \overline{2} / 033 / \overline{4} \overline{1} \overline{1}$.

with $s_{L}>0$, and 12 points were used for the other side. The corresponding numbers of $\omega / 2 \theta$ measurements in this case are 13 and 35 , respectively. The calculated value of the ratio $\Delta \bar{I} / \overline{\sigma(I)}$ is 6 . In both (c) and (d), the asymmetry is reversed rela- tive to $(a)$ and $(b), \bar{I}\left(s_{L}<0\right)<\bar{I}\left(s_{L}>0\right)$, hence $\Phi_{3}=\pi$ (negative triplet).

Of the 22 recorded profiles, 13 showed significant asymmetry corresponding to a unique triplet phase. In four other profiles the asym- 
metry was weaker and only a probable phase value could be assigned. Thus, 17 triplets were given a phase, i.e. $77 \%$ of the total. For the remaining 5 triplets the phase could not be determined. Among the 17 triplets, we determined 7 to be negative.

The 17 triplets were introduced with enhanced weights in a direct-method program. From the starting set thus generated, a phase model was developed which led directly to the correct structure. Later we found that 16 of the 17 experimental triplets were correct, the one in error belonging to the group of four with probable phase. Details of our application of experimental phase triplets in direct methods are given elsewhere. $^{30}$

\section{Conclusion}

We have shown that it is possible to estimate physically triplet phases for a centrosymmetric, mosaic crystal with unit cell volume $\sim 3000 \AA^{3}$ using standard $\mathrm{X}$-ray diffraction equipment, a four-circle diffractometer and filtered Mo radiation from a fine-focus tube. The incident beam was not monochromated by other means or focused.

The phase information was obtained from a careful mapping of the profiles of primary diffracted intensities during $\psi$ step-scans over preselected three-beam interactions. As predicted by theory, phase-related asymmetry was detected most easily for triplets with a relatively weak primary reflection $H$. However, significant effects were found also with strong $H$. In one case, $15 \overline{4}, F(\mathbf{H})$ is among the largest $1 \%$ of the data. Selection of triplets was based in part on the requirement that both the secondary, $L$, and coupling, $H-L$, reflections should belong to a group of the most intense reflections for the crystal. With this condition it was possible to assign phases to $77 \%$ of the triplets examined.

We have compared our new measurements with those made previously on a crystal of very similar mosaicity but with a smaller unit cell, $V \sim$ $684 \AA^{3}{ }^{4}$ In general, the signal/background ratio is much smaller in the present case. Nevertheless, the results clearly indicate that it should be possible to obtain phase information for similar crystals with larger unit cells than EHM III under the same experimental conditions as used here. From an applied point of view, it is a very encouraging result that phase measurements can be made as part of a data collection for crystals with unit cell volumes of at least $\sim 3000 \AA^{3}$. As discussed in preceding paragraphs, the physical conditions for such measurements can be refined and improved in various ways. We conclude, therefore, that there is a lot more potential in this method than has generally been realized before.

The experimental phases were used to solve an unknown organic structure with molecular weight 298.3 and with 22.5 non-hydrogen atoms in the asymmetric unit. The crystal has 8 molecules per cell $(Z=8)$. We observe that a primitive triclinic crystal with similar unit cell volume and density, and with $Z=2$ would contain a structure of molecular weight $\sim 1200$ and with approximately 90 non-hydrogen atoms.

We may sum up some of the advantageous features of the physical method for phase acquisition:

(a) Negative triplets are as accessible to measurement as positive ones. In fact, since triplets with a weak $H$, and strong $L$ and $H-L$ reflections are the most favourable for measurement, one may argue on statistical grounds that negative triplets are generally easier to measure. These phase relations may be used to great advantage in the initial stages of a structure solution.

(b) Experimental triplets with a common $H$ reflection may be combined in quartets or higher $n$-tuplets.

(c) Measured phases may become particularly useful in work with structures possessing pseudotranslation symmetry. Such structures, even of moderate size, may be exceedingly difficult to solve both by Patterson and standard direct methods. The reflection data are frequently characterized by a large fraction of aberrant triplets and by isolated groups of reflections which interact very little or late in the normal $\Sigma_{2}$ process. Knowledge of the phases for these reflections may be essential for unravelling the structure of the non-regular part of the molecule. Isolated reflection-groups can be identified from an analysis of the data, and triplets bridging these islands with the bulk of reflections may then be selected for measurements.

We are preparing to carry out work on structures with pseudo-translation symmetry, in order to study the possible usefulness of experimental phases in such cases. 
Acknowledgement. This work was supported in part by Grant D.22.52.005 from Norges Almenvitenskapelige Forskningsråd (NAVF).

\section{References}

1. (a) Miyake, S. and Kambe, K. Acta Crystallogr. 7 (1954) 220; (b) Kambe, K. Acta Crystallogr. 7 (1954) 777; (c) Kambe, K. J. Phys. Soc. Jpn. 12 (1957) 13.

2. Hart, M. and Lang, A. R. Phys. Rev. Lett. 7 (1961) 120.

3. Post, B. Phys. Rev. Lett. 39 (1977) 760.

4. Thorkildsen, G. and Mo, F. Abstracts of the 7th European Crystallographic Meeting (ECM-7), Jerusalem 1982, p. 6.

5. Thorkildsen, G. and Mo, F. Abstracts of the 8th European Crystallographic Meeting (ECM-8), Liège 1983, p. 258.

6. Chang, S. L. Phys. Rev. Lett. 48 (1982) 163.

7. Gong, P. P. and Post, B. Acta Crystallogr., Sect. A39 (1983) 719.

8. Mo, F., Thorkildsen, G. and Hauback, B. C. $A b$ stracts of Symposium on Precision and Molecular Structures, Seattle, June 2-4 1986, p. 4.

9. Chang, S. L. Crystallogr. Rev. 1 (1987) 87.

10. Bremer, J. and Thorkildsen, G. Acta Crystallogr., Sect. A 42 (1986) 191.

11. Thorkildsen, G. Acta Crystallogr., Sect. A43 (1987) 361 .

12. Batterman, B. W. and Cole, H. Rev. Mod. Phys. 36 (1964) 681.

13. von Laue, M. Röntgenstrahlinterferenzen, Akademische Verlagsgesellschaft, Leipzig 1941, Chap. 5.

14. Bethe, H. A. Ann. Phys. (Leipzig) 87 (1928) 55.
15. Marthinsen, K. Hovedoppgave, University of Trondheim-NTH, Trondheim, Norway 1981.

16. Thorkildsen, G. Doctoral Thesis, University of Trondheim-NTH, Trondheim, Norway 1983.

17. Thorkildsen, G. and Mo, F. In preparation.

18. Hümmer, K. and Billy, H. Acta Crystallogr., Sect. A 38 (1982) 841.

19. (a) Juretschke, H. J. Phys. Rev. Lett. 48 (1982) 1487; (b) Juretschke, H. J. Phys. Lett. A 92 (1982) 183; (c) Juretschke, H. J. Acta Crystallogr., Sect. A 40 (1984) 379.

20. Høier, R. and Marthinsen, K. Acta Crystallogr., Sect. A 39 (1983) 854.

21. Hümmer, K. and Billy, H. Acta Crystallogr., Sect. A 42 (1986) 127.

22. Ladell, J. Am. Crystallogr. Assoc. Program Abstr., Ser. 2, 10 (1982) 40.

23. (a) Nicolosi, J. Am. Crystallogr. Assoc. Program Abstr., Ser. 2, 10 (1982) 39; (b) Post, B., Nicolosi, J. and Ladell, J. Acta Crystallogr., Sect. A40 (1984) 684.

24. Chang, S. L. Phys. Rev. B 33 (1986) 5848.

25. James, R. W. The Optical Principles of the Diffraction of X-Rays; The Crystalline State, Bell, London 1948, Vol. 2, pp. 34-46.

26. Heinrich, K. F. J. In: McKinley, T. D., Heinrich, K. F. J. and Wittry, D. B., Eds., The Electron Microprobe, Wiley, New York 1966, pp. 296-377.

27. Han, F. S. and Chang, S. L. Acta Crystallogr., Sect. A39 (1983) 98.

28. Busing, W. R. and Levy, H. A. Acta Crystallogr. 22 (1967) 457.

29. Tanaka, K. and Saito, Y. Acta Crystallogr., Sect. A 31 (1975) 841.

30. Hauback, B. C. and Mo, F. Acta Chem. Scand., Ser. A 42 (1988). 139.

Received October 19, 1987. 